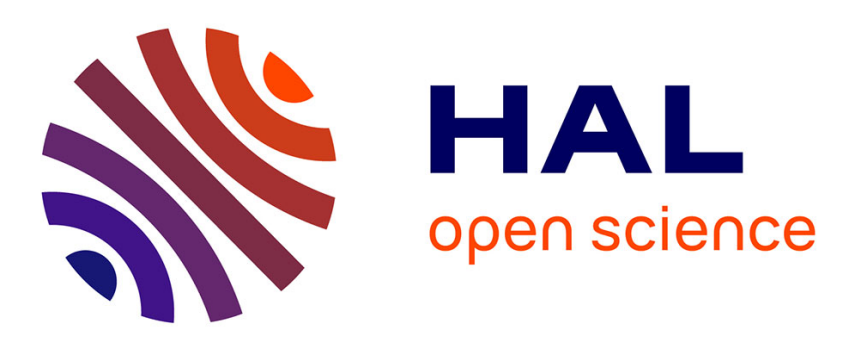

\title{
A non-invasive methodology for ATAA rupture risk estimation
}

O. Trabelsi, M Gutierrez, Sedigheh Farzaneh, A. Duprey, S. Avril

\section{To cite this version:}

O. Trabelsi, M Gutierrez, Sedigheh Farzaneh, A. Duprey, S. Avril. A non-invasive methodology for ATAA rupture risk estimation. Journal of Biomechanics, 2017. hal-01671296

\section{HAL Id: hal-01671296 https://hal.science/hal-01671296}

Submitted on 22 Dec 2017

HAL is a multi-disciplinary open access archive for the deposit and dissemination of scientific research documents, whether they are published or not. The documents may come from teaching and research institutions in France or abroad, or from public or private research centers.
L'archive ouverte pluridisciplinaire HAL, est destinée au dépôt et à la diffusion de documents scientifiques de niveau recherche, publiés ou non, émanant des établissements d'enseignement et de recherche français ou étrangers, des laboratoires publics ou privés. 
2

3

4

5

6

7

8

9

10

11

12

13

14

15

16

17

18

19

20

21

22

23

24

25

26

27

28

29

30

31

32

33

34

35

36

37

38

39

40

41

\section{A non-invasive methodology for ATAA rupture risk estimation}

O. Trabelsi ${ }^{* 1}$, M. Gutierrez ${ }^{1}$, S. Farzaneh ${ }^{1}$, A. Duprey ${ }^{1,2}$, S. Avril ${ }^{1}$

${ }^{1}$ Mines Saint-Étienne, CIS-EMSE, F-42023 Saint-Étienne, France. INSERM, U1059, SAINBIOSE, F-42023 Saint-Étienne, France. Université de Lyon, F-69000 Lyon, France.

${ }^{2}$ Hôpital Nord, Cardiovascular Surgery Service, CHU de Saint-Étienne, F-42055 SaintÉtienne, France.

*Corresponding author.

OIfa TRABELSI

Tel.: +33 695043825/+33 477420005

E-mail address: olfa.trabelsi@emse.fr 
43

44

45

46

47

48

49

50

51

52

53

54

55

56

57

58

59

60

Ascending thoracic aortic aneurysms (ATAA) are a life-threatening pathology provoking an irreversible dilation with a high associated risk of aortic rupture or dissection and death of the patient. Rupture or dissection of ATAAs remains unpredictable and has been documented to occur at diameters less than $4.5 \mathrm{~cm}$ for nearly $60 \%$ of patients. Other factors than the aneurysm diameter may highly affect the predisposition to rupture. In order to have a better insight in rupture risk prediction, a bulge inflation bench was developed to test ATAAs samples collected on patients during surgical interventions. Preoperative dynamic CT scans on a cohort of 13 patients were analyzed to estimate volumetric and cross-sectional distensibility. A failure criteria based on in vitro ultimate stretch showed a significant correlation with the aortic membrane stiffness deduced from in vivo distensibility. These results reinforce the significance of stretch-based rupture criteria and their possible noninvasive prediction in clinical practice.

Keywords: material property, ascending thoracic aortic aneurysm, brittleness, risk of rupture.

Word count: 3357 


\section{INTRODUCTION}

Ascending thoracic aortic aneurysm (ATAA) is generally an asymptomatic localized enlargement of the aortic diameter which makes it difficult to detect. Untreated or undetected ATAAs can lead to instantaneous death caused by dissection or rupture of the aneurysm (Johanson et al., 1995, Ramanath et al., 2009). In population-based studies, the annual incidence of aortic dissection ranged from 6 cases per 100,000 in a British study, to 9.1 per 100,000 for women and 16.3 per 100,000 for men in a Swedish study (Goldfinger et al., 2014).

Concerning ATAA, timely surgery is required; it consists in replacing the diseased aortic segment with a synthetic graft. Elective surgical repair of ATAAs is recommended for diameters larger than $5.5 \mathrm{~cm}$ or for fast growing aneurysms $(>1 \mathrm{~cm}$ per year) for patients without any familial disorders such as Marfan syndrome (Elefteriades et al., 2010). The diameter of $5.5 \mathrm{~cm}$ as a criterion for deciding surgical repair is widely acknowledged as insufficient. For instance, the International Registry of Acute Aortic Dissection (IRAD) reported that among 591 type "A" aortic dissections, 59\% had a diameter below $5.5 \mathrm{~cm}$ (Pape et al., 2007). Several studies dedicated to abdominal aortic aneurysms indicate that biomechanical factors may better predict the risk of rupture than the diameter criterion (Fillinger et al., 2003, McGloughlin, 2011, Leemans et al., 2016).

Biomechanical studies have also been achieved to have better insights in ATAAs rupture or dissection (Vorp et al., 2003, Pasta et al., 2016, 2012) and to elucidate the risk profile of the thoracic aorta (Martufi et al., 2016, Trabelsi et al., 2015). Recently, Trabelsi et al. (2016) developed an approach to identify the patient-specific material properties of ATAAs by minimization of the difference between model predictions and gated CT images. Moreover, they characterized the mechanical properties of ATAA on collected samples of patients undergoing surgical repair. Our research group (Duprey et al, 2016) also defined a rupture risk indicator based on the brittleness of the tissue (the rupture criterion is reached when the stretch applied to the tissue is greater than its maximum extensibility or distensibility) and showed a strong correlation between this rupture risk criterion and the physiological elastic modulus of ATAAs estimated within a bulge inflation test. The elastic properties of the aorta, responsible of the Windkessel effect, may change significantly with age or in pathological conditions. The determination of these properties may give an insight toward abnormalities undetectable on aortogram or echocardiogram and may predict the evolution of some diseases (Stefanadis et al., 1990).

Aortic distensibility is an accurate and reproducible parameter closely related to the bioelastic function of the aorta, and can serve as a marker to identify early cardiovascular diseases (Voges et al., 2012, Redheuil et al., 2010). It can be measured from changes in aortic diameter and pulse pressure. The aortic diameter can be measured non-invasively using gated cine-MRI and echocardiographic or invasively using angiographic techniques (Cavalcante et al., 2011, Wilson et al., 2003, Stefanadis et al., 1990). This parameter has been presented and analyzed in several previous studies. It has been studied for healthy subjects (Voges et al., 2012, Stefanadis et al., 1990), patients with Marfan's syndrome (Adams et al., 1995) or coronary artery diseases (Stefanadis et al., 1990), ascending/descending thoracic aortic aneurysms (Koullias et al., 2005, Cavalcante et al., 2011, Redheuil et al., 2010, Stefanadis et al., 1990), and infrarenal abdominal aortic aneurysms (Wilson et al., 2003). It has been considered by Redheuil et al (2010), as the most sensitive and specific marker of age-related arterial stiffness and dysfunction of large artery in individuals less than 50-year-old.

All cited studies have reported that the distensibility can be a reliable measurement related to changes in aortic wall structure, aneurysm growth and rupture. 
The main objective of this study is to show that the rupture risk of ATAAs could be predicted non-invasively using preoperative dynamic imaging. After acquiring gated CT images on 13 patients and after using them to estimate the aortic distensibility, we show that the aortic membrane stiffness deduced from this distensibility is well correlated with a recently proposed stretch-based rupture risk.

\section{MATERIAL AND METHOD}

This study involves a cohort of 13 patients who underwent elective surgery for ATAA repair at the University Hospital of Saint-Etienne (CHU-SE). Patient records were reviewed to obtain demographic data, medical history, and blood pressure information (Table 1).

Neither diabetes nor infections were present in the cohort. One of the patients (Patient 9) had the Marfan syndrome.

Following IRB agreement and after informed consent, a pre-operative ECG gated dynamic CT scan was acquired for each patient. The scans were processed to reconstruct the thoracic aorta and aneurysm geometries during the cardiac cycle, including diastole and systole. For each patient, CHU-SE supplied DICOM images of 10 phases throughout the cardiac cycle (resolution: $512 \times 512$, slice thickness $=0.5 \mathrm{~mm}$ ). The lumen of the aneurysm was clearly visible in the DICOM files, but detection of the aneurysm surface was not possible automatically.

A non-automatic segmentation of the CT image slices was performed using MIMICS (v. 10.01, Materialise NV). The three-dimensional surface of the aorta in each phase was identified and the aneurysm recognized. The three-dimensional (3D) surface of aorta was generated for each phase and exported in STL format. Smoothing factor for all phases was assumed identical. To recognize the systolic and diastolic phases, the luminal volumes of all phases were calculated. The systolic scan was defined as the one with the largest volume and the diastolic scan as the one with the smallest volume. Diastolic and systolic peripheral arterial pressure was obtained by cuff sphygmomanometry before the CT scan.

The reconstructed surfaces were imported in Rhinoceros (v.4.0, Robert McNeel \& Associates) to measure the changes of cross-sectional areas of the ascending and descending thoracic aorta, and the changes of volume of the aortic lumen across the whole ascending aorta, throughout the cardiac cycle. The luminan area was determined by defining a cross section perpendicular to the centerline and measuring its intersection with the segmented aorta using the commercial package MIMICS. The volume was determined as the total volume occupied by luminal voxels located between the cross section taken right after the right coronary artery and the one taken right before the brachiocephalic artery.

The cross-sectional distensibility $D_{A}\left(\mathrm{mmHg}^{-1}\right)$ was then estimated using the following formula:

$$
D_{A}=\frac{A_{\max }-A_{\min }}{A_{\min } *\left(P_{s y s}-P_{\text {dia }}\right)}=\frac{\Delta A}{A \Delta P}
$$

where $A_{\max }$ and $A_{\min }$ represent the maximal and minimal cross-sectional area of the aortic geometry $\left(\mathrm{mm}^{2}\right)$, and $P_{s y s}$ and $P_{\text {dia }}$ represent the systolic and diastolic blood pressure $(\mathrm{mmHg})$, respectively.

We also introduced the segmental volume distensibility $D_{V}\left(m m \mathrm{Hg}^{-1}\right)$, 


$$
D_{V}=\frac{V_{\max }-V_{\min }}{V_{\min } *\left(P_{s y s}-P_{\text {dia }}\right)}=\frac{\Delta V}{V \Delta P}(\text { eq. 2) }
$$

157

where $V_{\max }$ and $V_{\min }$ represent the maximal and minimal volumes of the ascending or descending aortic lumen $\left(\mathrm{mm}^{3}\right)$. This value was estimated only for the aneurysmal part of the ascending aorta.

After assessing aortic distensibility, we used the Laplace law to define the tangent membrane stiffness in the circumferential direction, named $E_{\text {in vivo, }}$, as:

$$
E_{\text {in vivo }}=\frac{\phi}{D} \quad \text { (eq. 4) }
$$

where $\phi$ is the diameter of the aneurysm measured from the CT scans. $D$ is the distensibility. Although $D$ is commonly defined using the cross-sectional area variation (in this case denoted $D_{A}$ ), volume variation were used to estimate $E_{\text {in vivo }}$ in the present study (in this case denoted $\left.D_{V}\right)$. A statistical study was performed to compare $\left(D_{A}\right)$ and $\left(D_{V}\right)$. A parametric inference was assumed comparing the relative volume variation $\Delta \mathrm{V} / \mathrm{V}$ and the relative cross-sectional area variation for the ascending and descending aorta $\Delta \mathrm{A} / \mathrm{A}$. The normal distribution was verified by the Anderson-Darling test. The analysis of variance (ANOVA) was implemented to evaluate the influence percentage of these factors. Those statistical methods were performed using Minitab ${ }^{\circledR}$.

As a membrane stiffness, $E_{\text {in vivo }}$ has the dimension of a stress per unit thickness. As there is no means of measuring accurately the thickness of the aortic wall in vivo, membrane stiffness was assessed instead of elastic or Young modulus.

Additionally to the ECG gated dynamic CT scans, tissue samples were obtained from the 13 patients after ATAA surgical repair. The samples were stored in saline solution at $4^{\circ} \mathrm{C}$ until mechanical testing within 24 hours. Immediately before testing, the thickness of each ATAA was measured at 10 locations; the average thickness $h_{0}$ for each patient is reported in Table 2 . All the collected samples were tested in bulge inflation, during which images were recorded and processed using a stereo Digital Image Correlation (sDIC) system to reconstruct the 3D shape and deformations of the inflated samples. The experimental set-up and the bulge inflation tests were explained in details in previous publications (Trabelsi et al., 2015, Romo et al., 2014, Davis et al., 2015).

We defined a tangent membrane stiffness, named $E_{\text {in vitro }}$, as:

Where:

$$
E_{\text {in vitro }}=\lambda_{1, \text { physio }} \frac{d \tau_{1, \text { physio }}}{d \lambda_{1, \text { physio }}} \text { (eq.5) }
$$

- $\tau_{1}$ is the circumferential component of the Kirchhoff stress tensor (the Kirchhoff stress is the Cauchy stress times the thickness, also named the tension by engineers);

- $\quad \lambda_{1}$ is the circumferential component of the stretch tensor;

- $\frac{d \tau_{1}}{d \lambda_{1}}$ is the slope of the circumferential stress/stretch curve;

- $\quad \tau_{1, \text { physio }}=\left(\tau_{1, \text { sys }}+\tau_{1, \text { dia }}\right) / 2$, where $\tau_{1 \text {,dia }}$ is the value taken by $\tau_{1}$ at diastole and $\tau_{1, \text { sys }}$ is the value taken by $\tau_{1}$ at systole; 
- $\quad \lambda_{1, \text { physio }}$ is the value taken by $\lambda_{1}$ when $\tau_{1}=\tau_{1, \text { physio }} . \lambda_{1, \text { physio }}$ is the circumferential stretch occurring in the aorta for a deformation between $P=0$ and $P=\mathrm{P}_{\text {physio }}$; where: $P_{\text {physio }}=\frac{P_{\text {sys }}+P_{\text {dias }}}{2}$.

$\tau_{1, \text { dia }}$ and $\tau_{1, \text { sys }}$ were determined using the following equation based on the Laplace's law:

$$
\tau_{1, \text { dias } / \text { sys }}=\frac{P_{\text {dia } / \text { sys }} * \phi}{2} \quad \text { (eq.6) }
$$

From the bulge inflation tests, we also deduced the circumferential stretch at burst (rupture), named $\lambda_{1, \text { burst }}$. The risk of rupture, denoted $\gamma_{\text {stretch }}$, was retrospectively derived for each patient as the ratio between $\lambda_{1, \text { physio }}$ and $\lambda_{1, \text { burst }}$. When $\gamma_{\text {stretch }}$ is close to one, the specimen is close to rupture. All the samples had a rupture stretch larger than the stretch at systole (Duprey et al., 2016).

Relationships among different ways of estimating the membrane stiffness (volumetric distensibility, cross section distensibility, bulge inflation tests), was assessed using Pearson correlation analysis. A normality test was performed using Anderson-Darling test for the parametric inference, to determine whether the membrane stiffness of all patients estimated using each technique followed a normal distribution.

One-Way ANOVA was performed to define the statistical differences between methods. The results are presented as mean \pm typical error. The statistical significance was set at $p<0.05$. The applied statistical methods were calculated using Minitab ${ }^{\circledR}$.

The objective of the present study is to evaluate the correlation between $\gamma_{\text {stretch }}, E_{\text {in vitro }}$ and $E_{\text {in vivo. }}$.

\section{RESULTS}

A statistical analysis revealed that $E_{\text {in vitro }}, E_{\text {in vivo }}$ estimated using $D_{V}$ or $D_{A}$, and $\gamma_{\text {stretch }}$, have a normal distribution for a pre-determined level of significance of $95 \%(\mathrm{p} \geq 0.05)$ $\left(\mathrm{p}\left(E_{\text {in vitro }}\right)=0.103 ; \quad \mathrm{p}\left(E_{\text {in vivo }} \quad\right.\right.$ using $\left.\quad D_{V}\right)=0.081 ; \quad \mathrm{p}\left(E_{\text {in vivo }}\right.$ using $\left.D_{A}\right)=0.332 ;$ $\left.\mathrm{p}\left(\gamma_{\text {stretch }}\right)=0.395\right)$.

It also showed a non-significant difference between all membrane stiffness values, whether estimated in vitro ( $\left.E_{\text {in vitro }}\right)$ or estimated in vivo using the distensibility $\left(E_{\text {in vivo }}\right)(\mathrm{p}=0.05, \mathrm{~S}$ $=2.577, \mathrm{R}^{2}=16.69 \%$ ). This means that the membrane stiffness of the ATAA is independent from the way of estimating it.

Distensibility values were calculated and analyzed for both ascending and descending thoracic aorta, using volume, and cross section variations of the aorta during the cardiac cycle (Figure $1)$.

To verify tendencies published in the literature (Koullias et al., 2005, Voges et al., 2012, Redheuil et al., 2010), distensibility variation with age and aneurysm diameter were plotted. Figure (2) confirms these tendencies and shows a decrease of the distensibility when age or maximal diameter of the aneurysm increases.

A plot of $E_{\text {in vitro }}$ versus $\gamma_{\text {stretch }}$ for the 13 patients of this study is displayed in Figure 3, showing that an exponential fit can give a coefficient of determination of 0.88 . 
By analyzing the volume and the cross-sectional area variation of the ascending aorta of all patients, it was shown that the values of distensibility estimated from the cross section area is generally smaller compared to distensibility estimated from volume variation (Figure 4-A). Moreover, by comparing the cross section area variation, it can be seen that it is always lower for the ascending aorta than for the descending one. Only patient 9 showed a different trend. In addition to BAV, patient 9 had a coarctation in the descending thoracic aorta (Figure 7-B). By comparing the ascending and the descending aorta, we showed that the ATA is stiffer than the DTA which is a direct consequence of the aneurysm formation.

Statistical study and data analysis showed that estimating the in vivo tangent physiological membrane stiffness by estimating the distensibility $\left(D_{V}\right)$, or by estimating the distensibility $\left(D_{A}\right)$ of the ascending aorta between systole and diastole, makes no significant differences.

The statistical analyses showed that a normal distribution was satisfied with a $\mathrm{p}$-value larger than 0.05 and that there is no significant difference between $\Delta \mathrm{V} / \mathrm{V}$ and $\Delta \mathrm{A} / \mathrm{A}$ in the ascending thoracic aorta (Figure 4-bis). Moreover, the data distribution of $\Delta \mathrm{V} / \mathrm{V}$ is less dispersed than the one of $\Delta \mathrm{A} / \mathrm{A}$ and it does not present any outlier (Figure 4-bis). For these reasons, $D_{V}$ was preferred to $D_{A}$ in the stiffness analysis. Results also showed that no difference exists in $\Delta \mathrm{A} / \mathrm{A}$ between the ascending and the descending thoracic aorta (Figure 4).

Results from patient 9 (with Marfan syndrome) were systematically excluded from the statistical study.

Figure 5 shows a linear correlation with a slope close to 1 between the in vitro tangent membrane stiffness and the in vivo membrane stiffness obtained using $D_{V}$.

This results leads to a direct relationship between the in vivo tangent membrane stiffness and the proposed index of rupture $\gamma_{\text {stretch }}$ (Figure 6). The intersection of the correlation between $D_{V}$ and $E_{\text {in vivo }}$ is around $3 \mathrm{MPa}$.

If we compare Figure 3 and Figure 6, an exponential tendency between $\gamma_{\text {stretch }}$ and both in vivo and in vitro tangential membrane stiffness of the aorta, with very similar fitting curves is shown. The coefficient of determination is smaller in Figure 6 than in Figure 3, with a coefficient of determination $\mathrm{R}^{2}=44 \%$ and $\mathrm{R}^{2}=88 \%$, respectively.

\section{DISCUSSION}

Our research group (Duprey et al., 2016) recently defined a novel rupture risk indicator, $\gamma_{\text {stretch }}$, based on the brittleness of the tissue (the rupture criterion is reached when the stretch applied to the tissue is greater than its maximum extensibility or distensibility) and showed a significant correlation between this rupture risk criterion and the physiological elastic modulus of ATAAs estimated within a bulge inflation test. In the present analysis, we show interestingly that $\gamma_{\text {stretch }}$ was also strongly correlated to the membrane stiffness of the ATAA that we assessed non-invasively from dynamic CT-scans by estimating the local aortic distensibility. This is a result with major significance as it means that the local aortic distensibility is a relevant measure to deduce the risk of rupture of ATAA. Other saying, the risk of rupture could be predicted in advance using measures of aortic distensibility.

Aortic distensibilities were reported by several authors, although none had shown previously that deducing the membrane stiffness could be relevant to evaluate the risk of rupture of ATAA. It is worth mentioning that aortic distensibilities estimated for the 13 patients of our study, whether based on cross section area or volume variations, showed similar tendencies as 
what was previously found in the literature. In fact, the distensibility decreases with age (see Figure 2), as demonstrated by Voges at al. (2012) and Redheuil et al. (2010). Distensibility decreases when aneurysmal diameter increases as published by Koullias et al. (2005) (see Figure 2).

The aorta, through its elastic properties, actively participates into the propulsion of the blood and interplays with the left ventricle in a "game of catch" with the stroke volume. A properly functioning aorta actually unloads the left ventricle (Koullias et al., 2005). Therefore, the loss of elasticity of the ascending thoracic aorta means a loss of function which may induce many clinical consequences such as diastolic LV dysfunction with dyspnea, predisposition to angina, and heart failure, and small vessel degeneration in brain and kidney with intellectual deterioration and renal failure (O'rourke et al., 2007).

We claim here that the loss of elasticity also increases the risk of rupture. To better understand this, rupture must be defined in terms of maximum stretch. Rupture of the aortic tissue is traditionally defined when the maximum stress that the tissue can withstand is reached. However, when we derived the maximum stress ratio between the stress applied to the tissue in vivo and its strength, we noted that most of the collected ATAA samples were far from rupture (Duprey et al., 2016). The stretch-based definition of rupture, which is equivalent, states that rupture occurs when the stretch applied to the tissue exceeds its maximum extensibility or distensibility. This way of defining rupture can be more physiologically meaningful as it is reported that aneurysm rupture or dissections often occur at a time of severe emotional stress or physical exertion (Martin et al., 2013). Such emotionally or physically stressful situations can induce significant changes of blood volumes in the aorta, making more compliant aneurysms less prone to rupture as they can sustain such changes of volume. Based on this analysis, Martin et al. also defined a similar criterion, named the diameter risk, which is the ratio between the current diameter of the aneurysm and the rupture diameter (Martin et al., 2013). Like us, they also showed that the diameter risk increases significantly with the physiological elastic modulus of the artery. Indeed, if the aortic wall is stiff, a rather large increase of pressure can be induced by a small increase of volume.

The main results of this study is accordingly the tendency found between $\gamma_{\text {stretch }}$ and $E_{\text {in vivo }}$, but this tendency shows a low $\mathrm{R}^{2}$. This result is still insufficient to use $\gamma_{\text {stretch }}$ in a clinical setting and propose it as a new criterion for aneurysm rupture prediction. Further exploration of rupture index based on stretch is needed before possible clinical application. This is probably due to several limitations that should be addressed. The major one is the fact that $E_{\text {in vivo }}$ was estimated globally and homogenously across the ascending thoracic aorta through its volume variations. There was a higher $\mathrm{R}^{2}$ for the tendency between $\gamma_{\text {stretch }}$ and $E_{\text {in vitro }}$, but $E_{\text {in vitro }}$ and $\gamma_{\text {stretch }}$ were both estimated locally on the small $30 \times 30 \mathrm{~mm}^{2}$ sample tested in the bulge inflation device. Our group is currently working on an inverse method to deduce the local membrane stiffness in vivo using dynamic CT scan (Bersi et al., 2016).

Another difference to notice between in vivo and in vitro behavior is that, despite the significant correlation between $E_{\text {in vivo }}$ and $E_{\text {in vitro }}, E_{\text {in vivo }}$ averagely overestimated $E_{\text {in vitro }}$ (Figure 6). A possible explanation is the contribution of the perivascular environment in vivo, which was not taken into account here to derive the in vivo membrane stiffness. 
335 Possible inter-individual variations of the perivascular effects may also contribute to lower $\mathrm{R}^{2}$ 336 tendency between $\gamma_{\text {stretch }}$ and $E_{\text {in vivo }}$.

337 We mostly focused on the circumferential membrane stiffness, derived from aortic 338 distensibility. The longitudinal extensibility of the ascending thoracic aorta would also be 339 interesting for characterizing the elastic properties of ATAAs. The longitudinal extensibility 340 of the ascending thoracic aorta is caused by the heart movement during the cardiac cycle 341 (Beller et al., 2004, García-Herrera et al., 2013). The change of length could be measured by 342 tracking the position of anatomical reference such as the sinotubular junction and the 343 brachiocephalic artery at every phase throughout the cardiac cycle (see Figure 7).

344 Finally, our rupture index is the ratio between the maximum hoop stretch and in vivo hoop 345 stretch of the aorta. We need to investigate further what would be the effect of the presence of 346 residual stresses on such rupture index based on stretch.

\section{CONCLUSION}

348 In this paper, ATAA rupture was defined as the time when the stretch applied to the tissue 349 exceeds its maximum extensibility or distensibility. We showed interestingly that the 350 membrane stiffness of ATAA, assessable non-invasively from dynamic CT-scans by 351 estimating the local aortic distensibility, is a relevant measure to deduce this stretch-based risk 352 of rupture. Other saying, the risk of rupture of an ATAA could be predicted in advance using measures of aortic distensibility. Despite the major interest of this result, it would be interesting in future studies to consider also the longitudinal extensibility of the ascending thoracic aorta to better characterize the elastic properties of ATAAs.

\section{CONFLICT OF INTEREST}

The authors have no conflicts of Interest to declare concerning the contents of this manuscript. 


\section{REFERENCES}

Adams, J.N., Brooks, M., Redpath, T.W., Smith, F.W., Dean J. Gray, J., Walton, S., Trent, R.J. 1995. Aortic distensibility and stiffness index measured by magnetic resonance imaging in patients with Marfan's syndrome. British heart journal, 73(3), 265-269.

Beller, C. J., Labrosse, M. R., Thubrikar, M. J., Robicsek, F. 2004. Role of aortic root motion in the pathogenesis of aortic dissection. Circulation, 109(6), 763-769.

Bersi, M. R., Bellini, C., Di Achille, P., Humphrey, J. D., Genovese, K., Avril, S. 2016. Novel Methodology for Characterizing Regional Variations in the Material Properties of Murine Aortas. Journal of biomechanical engineering, 138(7), 071005.

Cavalcante, J.L., Lima, J.A.C., Redheuil, A., Al-Mallah, M.H. 2011. Aortic Stiffness. Current understanding and future directions. Journal of the american college of cardiology, 57(14), 1511-1522.

Davis, F.M., Luo, Y., Avril, S., Duprey, A., Lu, J. 2015. Pointwise characterization of the elastic properties of planar soft tissues: application to ascending thoracic aneurysms. Biomech. Model. Mechanobiol, 14(5), 967-78.

Duprey, A., Trabelsi, O., Vola, M., Favre, J.P., Avril, S. 2016. Biaxial rupture properties of ascending thoracic aortic aneurysms. Acta Biomater, 42, 273-85.

Elefteriades, J.A., Farkas, E.A. 2010. Thoracic aortic aneurysm clinically pertinent controversies and uncertainties. J Am Coll Cardiol, 55(9), 841-857.

Fillinger, M.F., Marra, S.P., Raghavan, M.L., Kennedy, F.E. 2003. Prediction of rupture risk in abdominal aortic aneurysm during observation: wall stress versus diameter. J Vasc Surg, 37(4), 724-32.

García-Herrera, C. M., Celentano, D. J. 2013. Modelling and numerical simulation of the human aortic arch under in vivo conditions. Biomechanics and modeling in mechanobiology, 12(6), 1143-1154.

Goldfinger, J. Z., Halperin, J. L., Marin, M. L., Stewart, A. S., Eagle, K. A., Fuster, V. Thoracic Aortic Aneurysm and Dissection. Journal of the american college of cardiology, 64 (16), 1725-1739, 2014.

Johansson, G., Markstrm, U., Swedenborg, J. 1995. Ruptured thoracic aortic aneurysms: a study of incidence and mortality rates. J Vasc Surg, 21(6), 985-958.

Koullias, G., Modak, R., Tranquilli, M., Korkolis, D. P., Barash, P., Elefteriades, J. A. 2005. Mechanical deterioration underlies malignant behavior of aneurysmal human ascending aorta. Journal of thoracic and cardiovacsular surgery, 130(3), 677-683. 
Leemans, E.L., Willems, T.P., van der Laan, M.J., Slump, C.H., Zeebregts, C.J. 2016. Biomechanical indices for rupture risk estimation in abdominal aortic aneurysms, 24(2), 254 261.

Martin, C., Sun, W., Pham, T., Elefteriades, J. 2013. Predictive biomechanical analysis of ascending aortic aneurysm rupture potential. Acta biomaterialia, 9(12), 9392-9400.

Martufi, G., Forneris, A., Appoo, J.J., Di Martino, E.S. 2016. Is there a role for biomechanical engineering in helping to elucidate the risk profile of the thoracic aorta? The Annals of thoracic surgery, 101(1), 390-398.

McGloughlin, T. 2011. Biomechanics and mechanobiology of aneurysms. ISBN, 7.

O'rourke, M. F., Hashimoto, J. 2007. Mechanical factors in arterial aging: a clinical perspective. Journal of the American College of Cardiology, 50(1), 1-13.

Pasta, S., Phillippi, J.A., Tsamis, A., D’Amore, A., Raffa, G.M., Pilato, M., Scardulla, C., Watkins, S.C., Wagner, W.R., Gleason, T.G. 2016. Constitutive modeling of ascending thoracic aortic aneurysms using microstructural parameters. Medical engineering \& physics, 38(2), 121-130.

Pasta, S., Phillippi, J.A., Gleason, T.G., Vorp, D.A. 2012. Effect of aneurysm on the mechanical dissection properties of the human ascending thoracic aorta. The Journal of thoracic and cardiovascular surgery, 143(2), 460-467.

Pape, L.A., Tsai, T.T., Isselbacher, E.M., Oh, J.K., OGara, P.T., Evangelista, A., Fattori, R., Meinhardt, G., Trimarchi, S., Bossone, E. 2007. Aortic diameter $5.5 \mathrm{~cm}$ is not a good predictor of type a aortic dissection observations from the international registry of acute aortic dissection (irad). Circulation, 116(10),1120-1127.

Ramanath, V.S., Oh, J.K., Sundt, T.M.3rd, Eagle, K.A. 2009. Acute aortic syndromes and thoracic aortic aneurysm. Mayo Clin Proc, 84(5), 465-481.

Redheuil, A., Yu, W.C., Wu, C.O., Mousseaux, E., de Cesare, A., Yan, R., Kachenoura, N., Bluemke, D., Lima, J.A.C. 2010. Reduced ascending aortic strain and distensibility earliest manifestations of vascular aging in humans. Hypertension. 55(2), 319-26.

Romo, A., Badel, P., Duprey, A., Favre, J.P., Avril, S. 2014. In vitro analysis of localized aneurysm rupture. J. Biomech, 47(3), 607-616.

Stefanadis, C., Statos, C., Boudoulas, H., Kourouklis, C., Toutouzas, P. 1990. Distensibility of ascending aorta: comparison of invasive and non-invasive techniques in healthy men and in men with coronary artery disease. European Heart Journal, 11(11), 990-6.

Trabelsi, O., Davis, F.M., Rodriguez-Matas, J.F., Duprey, A., Avril, S. 2015. Patient specific stress and rupture analysis of ascending thoracic aneurysms. J Biomech, 48(10), 1836-43.

Trabelsi, O., Duprey, A., Favre, J.P., Avril, S. 2016. Predictive models with patient specific material properties for the biomechanical behavior of ascending thoracic aneurysms. Ann Biomed Eng, 44(1), 84-98. 
458 Vorp, D.A., Schiro, B.J., Ehrlich, M.P., Juvonen, T.S., Ergin, M.A., Griffith, B.P. 2003. Effect 459 of aneurysm on the tensile strength and biomechanical behavior of the ascending thoracic aorta. The Annals of thoracic surgery, 75(4), 1210-1214.

461

Voges, I., Jerosch-Herold, M., Hedderich, J., Pardun, E., Hart, C., Gabbert, D.D., Hansen, J.H., Petko, C., Kramer, H.H., Rickers, C. 2012. Normal values of aortic dimensions, distensibility, and pulse wave velocity in children and young adults: a cross-section study. J. of cardiovacsular magnetic resonance, 14, 14-77. relationship between aortic wall distensibility and rupture of infrarenal abdominal aortic aneurysm. Journal of vascular surgery, 37(1), 112-117. 
$471 \quad$ Tables

472 Table 1. Patient clinical information

\begin{tabular}{|c|c|c|c|c|}
\hline Patient Id & Sex/ Valve & Age (year) & $\Delta \mathbf{P}(\mathbf{k P a})$ & Aneurysm diameter $(\mathbf{m m})$ \\
\hline 1 & $\mathrm{M} / \mathrm{BAV}$ & 58 & 8.86 & 65 \\
\hline 2 & M/TAV & 78 & 5.34 & 51 \\
\hline 3 & $\mathrm{M} / \mathrm{BAV}$ & 61 & 11.5 & 52 \\
\hline 4 & M/TAV & 69 & 5.34 & 50 \\
\hline 5 & M/BAV & 70 & 5.8 & 51 \\
\hline 6 & M/TAV & 81 & 5.34 & 50 \\
\hline 7 & M/TAV & 84 & 13.4 & 55 \\
\hline 8 & M/TAV & 74 & 10.68 & 51 \\
\hline $9 *$ & $\mathrm{M} / \mathrm{BAV}$ & 27 & 8.66 & 50 \\
\hline 10 & M/TAV & 42 & 5.34 & 55 \\
\hline 11 & M/TAV & 81 & 5.34 & 52 \\
\hline 12 & F/TAV & 78 & 5.34 & 65 \\
\hline 13 & M/BAV & 57 & 5.34 & 55 \\
\hline \multicolumn{2}{|c|}{ Mean } & 66.2 & 7.4 & 54 \\
\hline \multicolumn{2}{|c|}{ STD } & 16.9 & 2.9 & 5.2 \\
\hline
\end{tabular}

473 BAV: Bicuspid aortic valve, TAV: Tricuspid aortic valve

$474 *$ Patient with genetic disorder (Marfan syndrome) 
Table 2: Geometric and biomechanical parameters measured for the 13 patients

\begin{tabular}{|c|c|c|c|c|c|c|c|c|c|c|}
\hline Patient & $\mathbf{h}_{0}(\mathbf{m m})$ & $\begin{array}{c}\mathbf{E}_{\text {in vitro }} \\
(\mathbf{m m} * \mathbf{M P a})\end{array}$ & $\gamma_{\text {stretch }}$ & $\Delta \mathbf{A} / \mathbf{A}(\mathbf{a s c})$ & $\Delta \mathrm{V} / \mathrm{V}(\mathbf{a s c})$ & $\Delta \mathrm{A} / \mathrm{A}(\mathrm{desc})$ & $\underset{\left(\mathbf{m m H g}^{-1}\right)}{\mathrm{D}_{\mathbf{V}}}$ & $\underset{\left(\mathbf{m m H g}^{-1}\right)}{\mathbf{D}_{\mathrm{A}}}$ & $\begin{array}{l}\mathbf{E}_{\text {in vivo }}\left(D_{\mathrm{V}}\right) \\
(\mathbf{m m} * \mathbf{M P a})\end{array}$ & $\begin{array}{l}\mathbf{E}_{\text {in vivo }}\left(\mathbf{D}_{\mathrm{A}}\right) \\
(\mathbf{m m} * \mathbf{M P a})\end{array}$ \\
\hline 1 & 2.10 & 1.216 & 0.87 & 0.080 & 0.08 & 0.160 & 0.055 & 0.001 & 7.199 & 7.199 \\
\hline 2 & 1.98 & 2.202 & 0.92 & 0.100 & 0.11 & 0.110 & 0.062 & 0.002 & 2.476 & 2.723 \\
\hline 3 & 2.74 & 0.922 & 0.88 & 0.110 & 0.13 & 0.120 & 0.043 & 0.001 & 4.600 & 5.436 \\
\hline 4 & 1.86 & $* 0.866$ & 0.84 & 0.160 & 0.21 & 0.290 & 0.060 & 0.004 & 1.271 & 1.669 \\
\hline 5 & 2.50 & 3.996 & 0.95 & 0.110 & 0.06 & 0.110 & 0.051 & 0.003 & 4.930 & 2.689 \\
\hline 6 & 2.72 & 1.237 & 0.84 & 0.090 & 0.10 & 0.190 & 0.041 & 0.002 & 2.670 & 2.967 \\
\hline 7 & 1.79 & 3.314 & 0.94 & 0.090 & 0.12 & 0.160 & 0.070 & 0.001 & 6.142 & 8.189 \\
\hline 8 & 1.90 & 3.437 & 0.97 & 0.059 & 0.13 & 0.103 & 0.068 & 0.001 & 4.190 & 9.185 \\
\hline 9 & 1.77 & $* 0.569$ & 0.81 & 0.210 & 0.22 & 0.170 & 0.061 & 0.003 & 1.968 & 2.062 \\
\hline 10 & 1.59 & 1.026 & 0.88 & 0.054 & 0.16 & 0.086 & 0.074 & 0.001 & 1.836 & 5.396 \\
\hline 11 & 1.76 & 5.752 & 0.96 & 0.039 & 0.02 & 0.075 & 0.073 & 0.001 & 11.570 & 7.193 \\
\hline 12 & 2.41 & 4.124 & 0.95 & 0.034 & 0.02 & 0.034 & 0.053 & 0.001 & $* * 17.355$ & 10.089 \\
\hline 13 & 2.35 & 1.271 & 0.89 & 0.073 & 0.11 & 0.098 & 0.050 & 0.002 & 2.557 & 3.999 \\
\hline Mean & 2.11 & 2.591 & 0.90 & 0.093 & 0.11 & 0.131 & 0.059 & 0.002 & 4.284 & 5.292 \\
\hline STD & 0.39 & 1.625 & 0.05 & 0.049 & 0.06 & 0.064 & 0.011 & 0.001 & 2.936 & 2.856 \\
\hline
\end{tabular}

*Atypical value (observation) **Statistical outlier 


\section{Figures}

Figure 1: Method for estimating aortic distensibility using cross-section area and volume variation between systole and diastole. A- Ascending and descending cross-section area variation between systole and diastole. B- Ascending volume variation between systole and diastole.

Figure 2: Sectional and volumetric ascending aorta distensibility variation respect to (A) age and (B) maximal aneurysmal diameter. A- Distensibility variation with age. B- Distensibility variation with aneurysm diameter.

Figure 3: Correlation between the index of $\gamma_{\text {stretch }}$ and the tangent elastic modulus $E_{\text {in vitro }}$

Figure 4: Distensibility estimation using volume or cross section area variation between systole and diastole. A- Ascending aorta volume and cross section area. B- Cross-sectional area variation between the ascending and the descending aorta.

Figure 4-bis: A statistical comparison between the relative volume variation for the ascending aorta $\Delta \mathrm{V} / \mathrm{V}$ (asc) and the relative cross-sectional area variation for the ascending and descending aorta $\Delta \mathrm{A} / \mathrm{A}$ (asc) and $\Delta \mathrm{A} / \mathrm{A}($ desc)

Figure 5: Correlation between the in vivo and in vitro tangent membrane stiffness.

Figure 6: Correlation between the in vivo tangent membrane stiffness and the index of rupture $\gamma_{\text {stretch. }}$

Figure 7: Movement of the aorta in a healthy subject and in an ATAA patient. A- Healthy aorta: Movement of the carotids and left subclavian artery between the systolic and diastolic phase. B-Marfan patient (Patient 9): Movement of the right coronary between the systolic (2) and diastolic (1) phase. A stenosis in the descending aorta is visible. 


$$
\text { ח }
$$



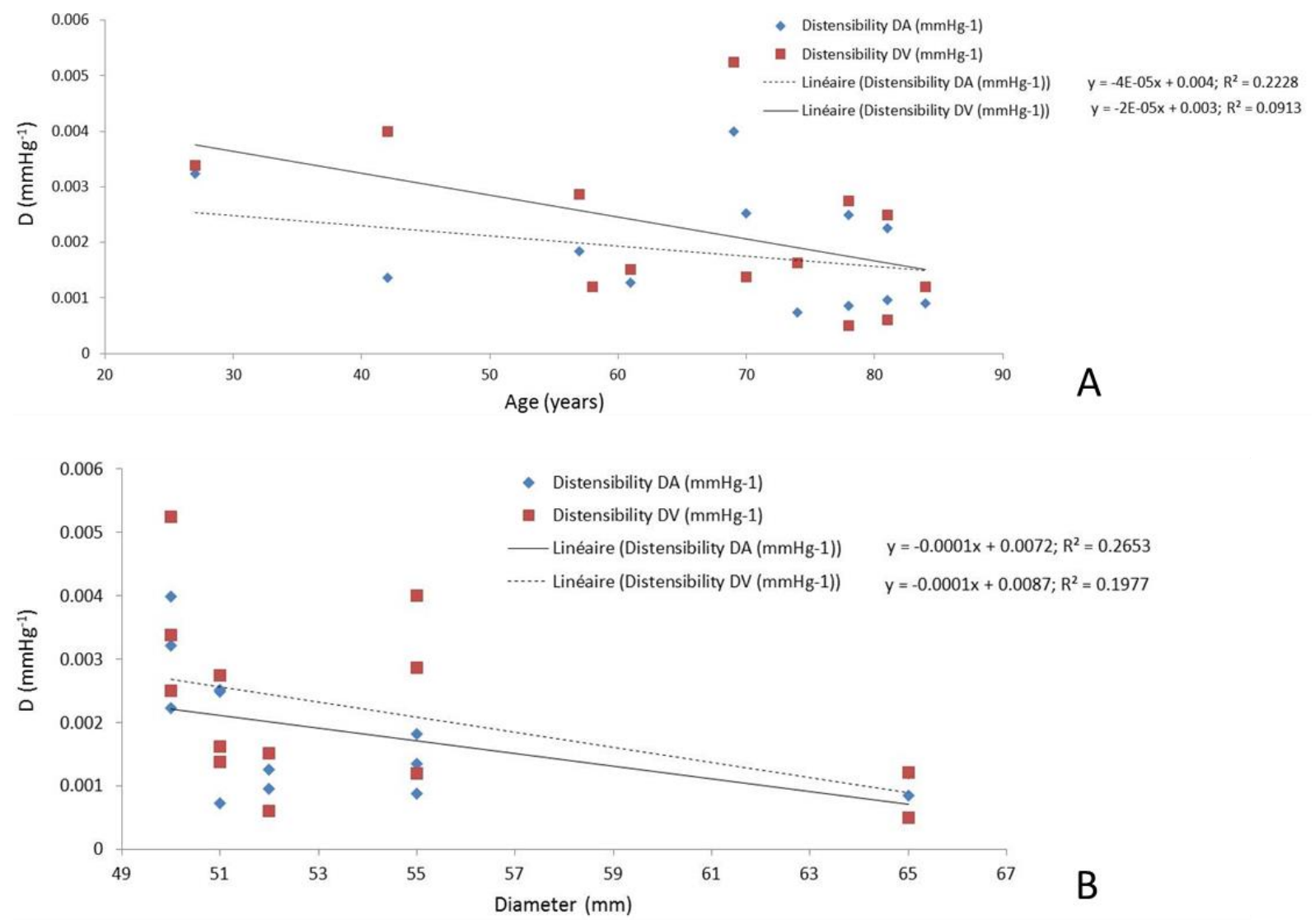


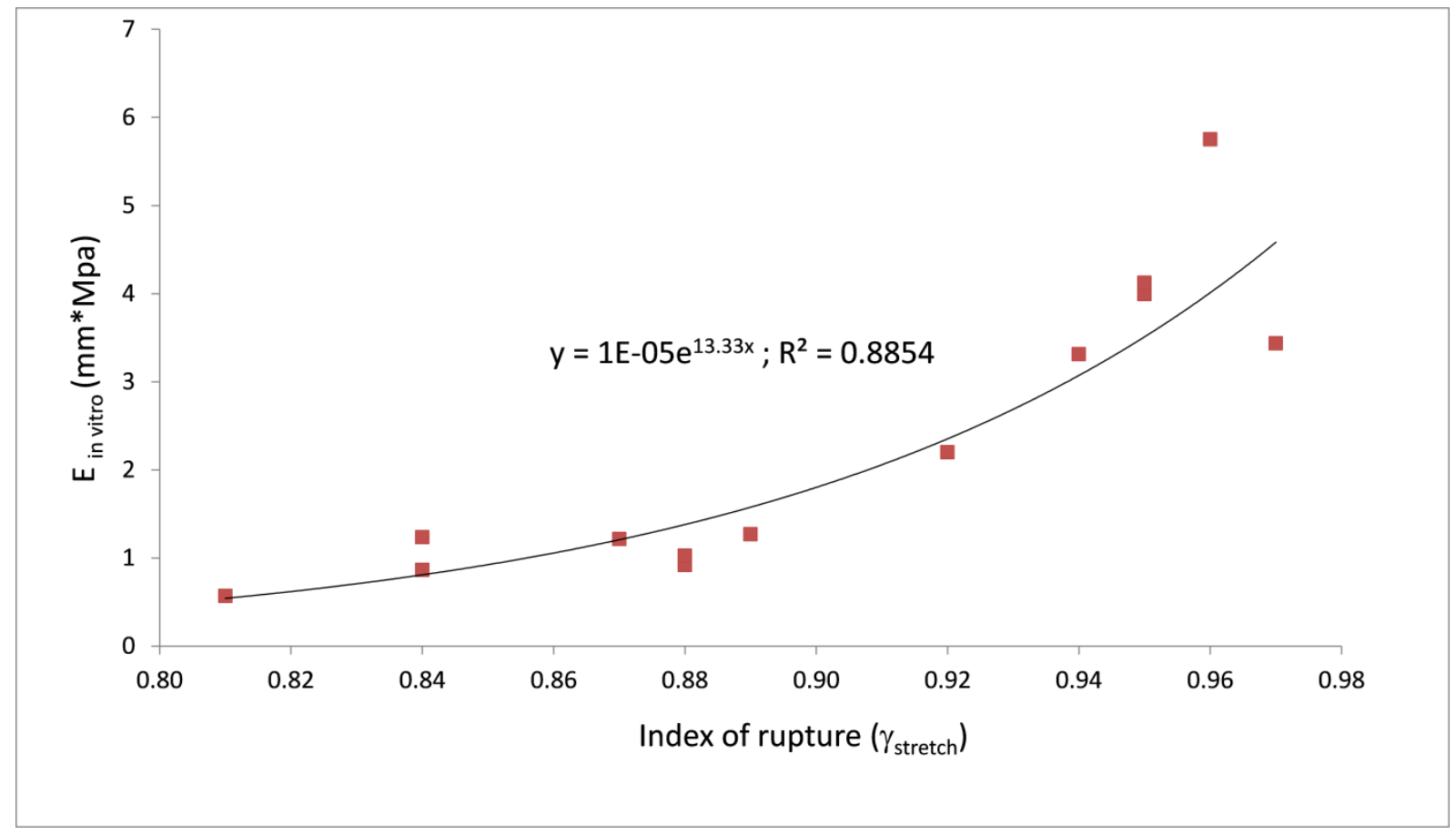




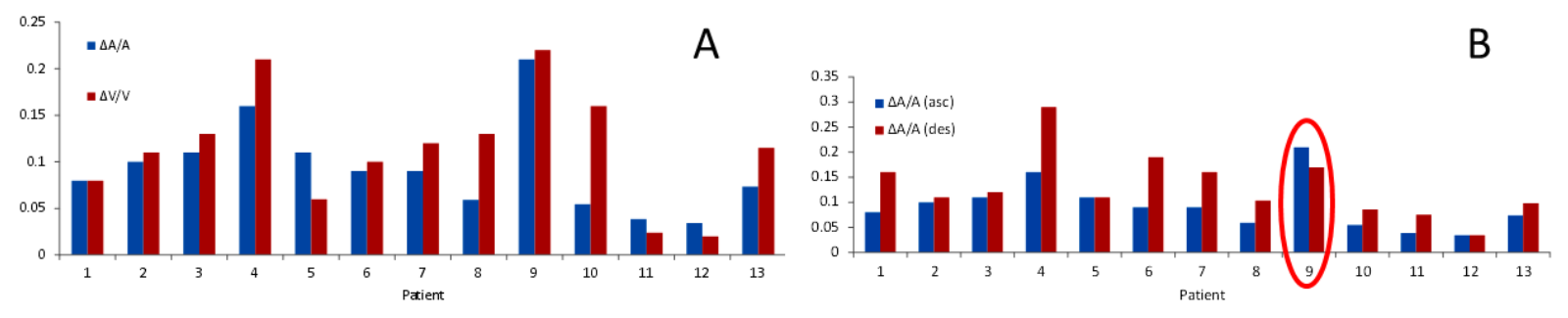



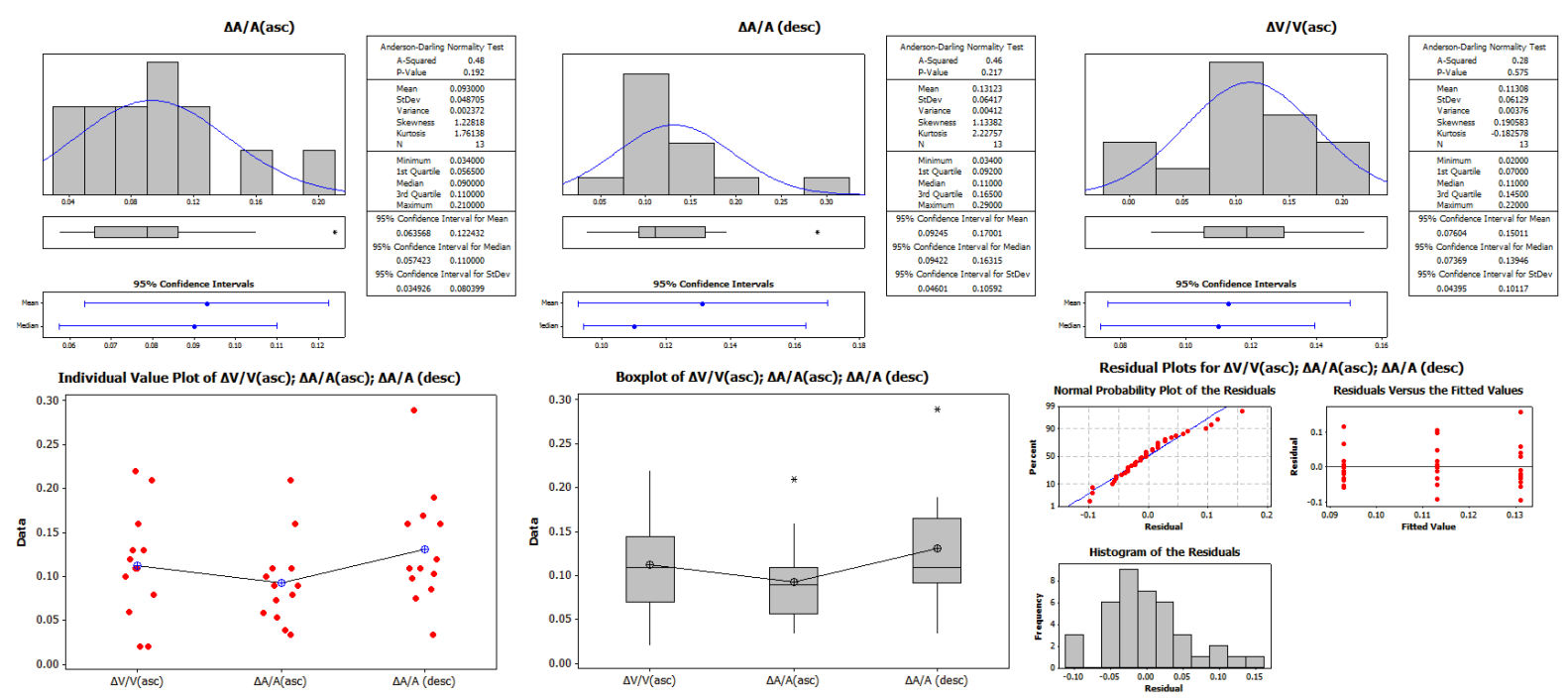


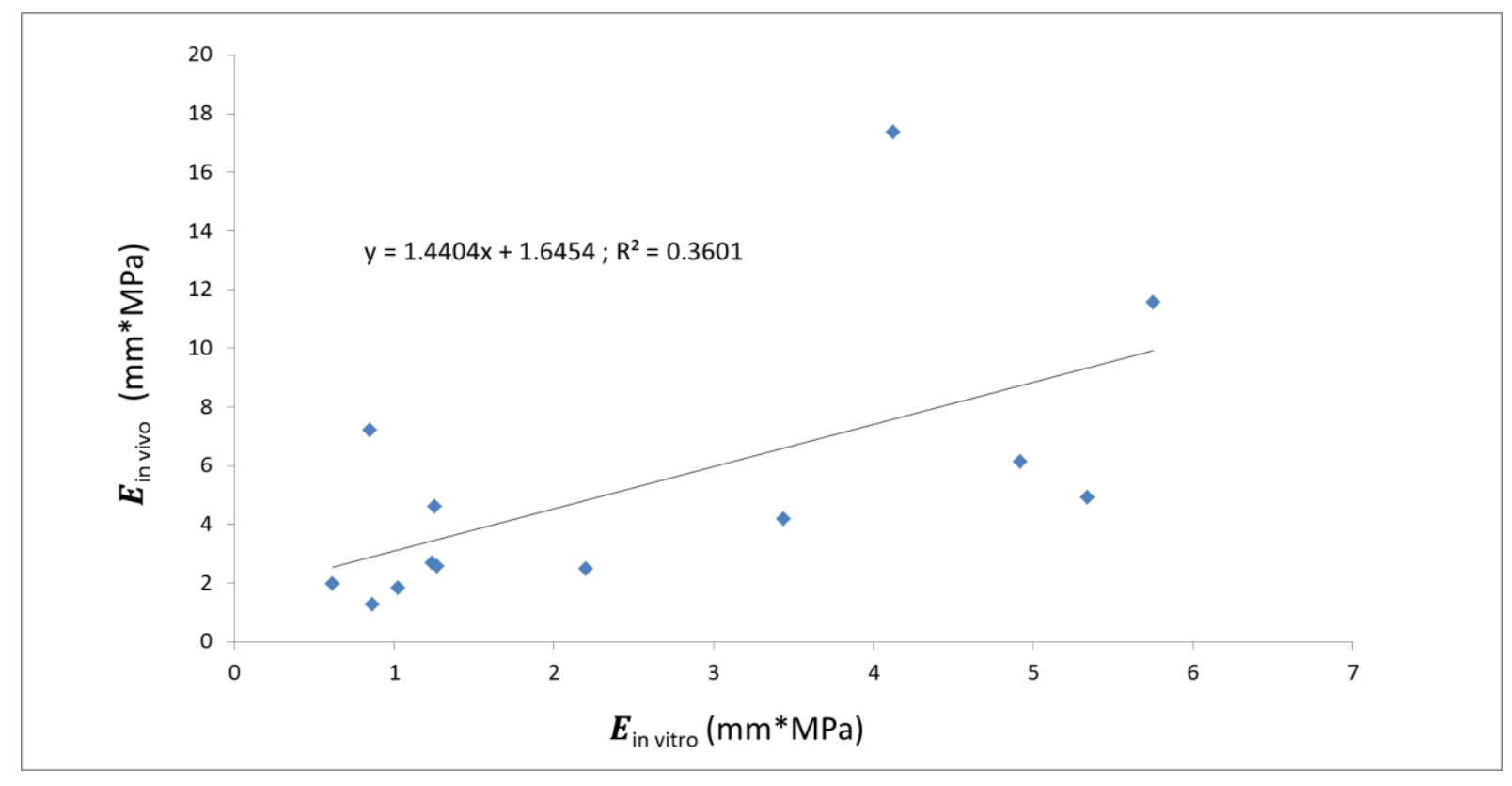




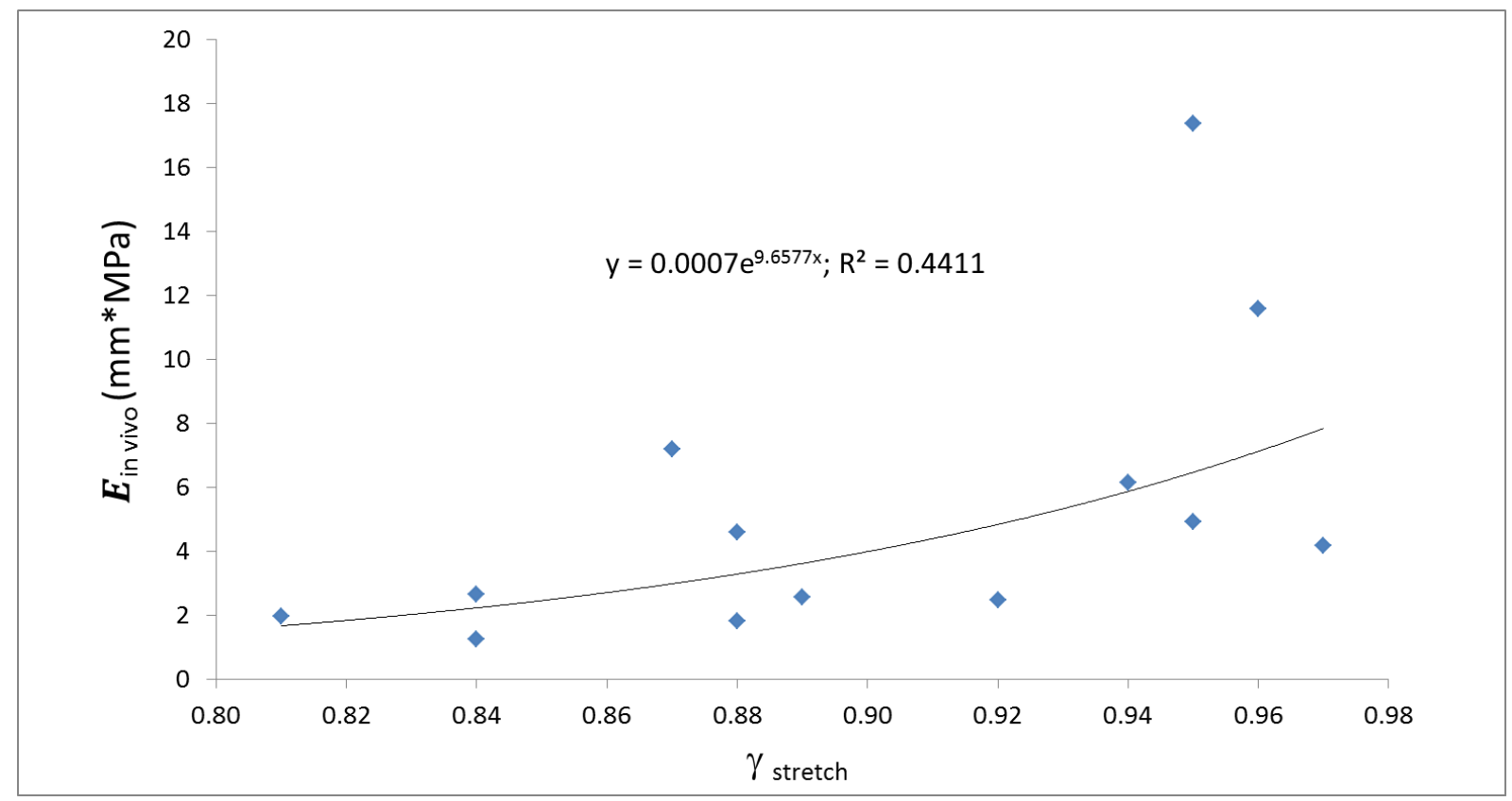




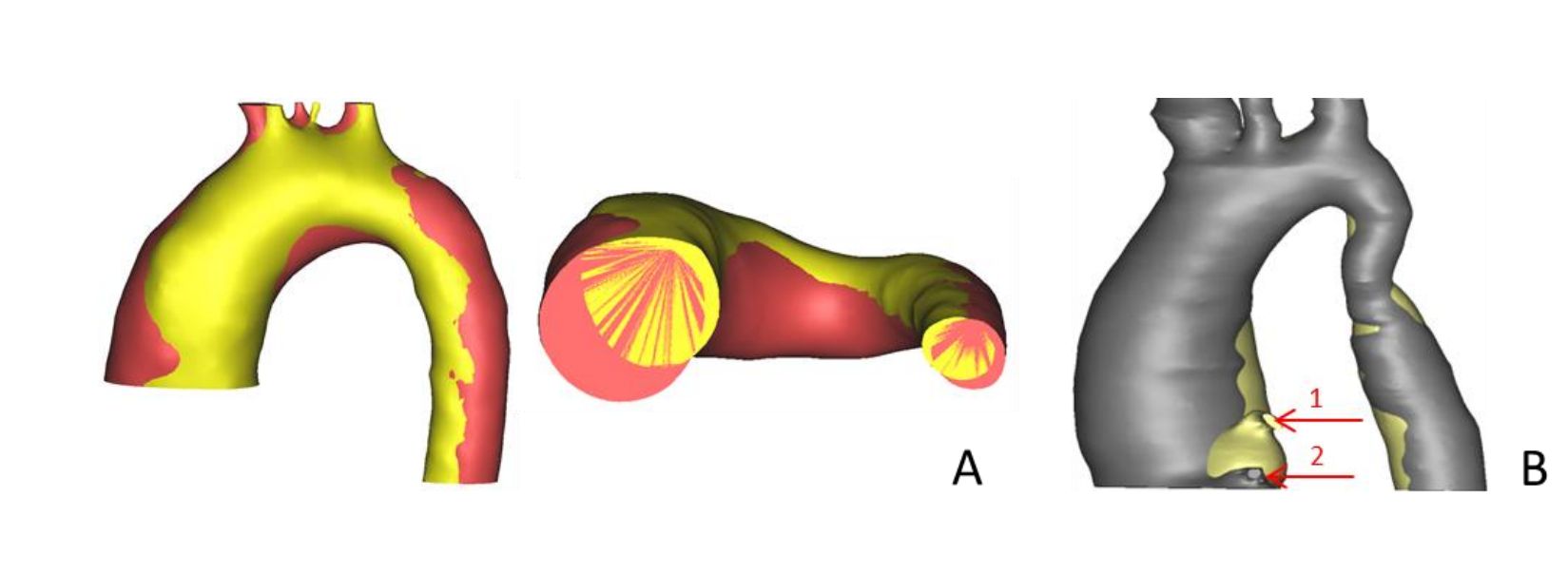

